\title{
ABELHAS (HYMENOPTERA: APOIDEA) VISITANTES DAS FLORES DA GOIABA EM POMAR COMERCIAL DE SALINAS, MG $\left(^{1}\right)$
}

\author{
ROSEMEIRE ALVES GUIMARÃES $\left({ }^{2}\right)$; RAQUEL PÉREZ-MALUF $\left({ }^{3 *}\right)$ \\ E MARIA APARECIDA CASTELLANI $\left({ }^{4}\right)$
}

\begin{abstract}
RESUMO
As abelhas são responsáveis por cerca de $80 \%$ a $100 \%$ da polinização de culturas agrícolas, especialmente aquelas relacionadas com a produção de sementes e frutos. A investigação da diversidade de abelhas em pomares de goiaba pode ser subsídio para estratégias de incremento da produtividade. Nesta perspectiva, o objetivo deste estudo foi identificar a diversidade de abelhas visitantes das flores de goiaba (Psidium guajava), em pomar comercial em Salinas (MG). O trabalho foi desenvolvido em maio de 2005 e foram coletadas as abelhas visitantes das flores nos horários entre $6 \mathrm{~h}$ e $18 \mathrm{~h}$, totalizando-se 44 horas de coleta. Coletaram-se 705 abelhas de 17 espécies, sendo Trigona spinipes a mais freqüente e dominante na cultura da goiaba. Apis mellifera, Melipona quadrifasciata e Tetragonisca angustula foram consideradas acessórias. Aproximadamente $84 \%$ dos indivíduos foram coletados da manhã, de $6 \mathrm{~h}$ às $10 \mathrm{~h}$.
\end{abstract}

Palavras-chave: Psidium guajava, estrutura de comunidade, visitantes florais.

\section{ABSTRACT \\ BEE DIVERSITY IN A COMMERCIAL GUAVA ORCHARD IN SALINAS, MINAS GERAIS STATE, BRAZIL}

Pollination is an important factor in agricultural systems, especially in growing fruits and seed production, which depend greatly on bee visiting during blossom season; highly successful gains within these activities varies between 80 and nearly 100 per cent, owing to the bees. The assessment of bee diversity in commercial orchards of guava may contribute to a more desirable strategic design and consequent improvement of production. The aim of the study was identify the diversity of visiting bees to guava flowers (Psidium guajava) in a commercial orchard in Salinas, State of Minas Gerais, Brazil. The work was carried during blossom season of May - 2005. Field works occurred between 6:00 am to 6:00 pm, counting with 44 hours of collection, when 705 bees were collected. The richness observed was of 17 species, the most frequent and dominant being Trigona spinipes. Among the collection there were some considered accessory species: Apis mellifera, Melipona quadrifasciata and Tetragonisca angustula. Most of individual bees have been captured during early periods of morning, between 6:00 and 10:00 am.

Key words: Psidium guajava, community structure, floral visitors, Apoidea

( ${ }^{1}$ ) Parte da dissertação apresentada ao Programa de Pós Graduação em Agronomia, da Universidade Estadual do Sudoeste da Bahia. Recebido para publicação em 29 de novembro de 2006 e aceito em 31 de julho de 2008

$\left({ }^{2}\right)$ Escola Agrotécnica Federal de Salinas (MG), Fazenda Varginha, km 02, Rodovia Salinas/Taiobeiras, Salinas (MG).

$\left({ }^{3}\right)$ Laboratório de Zoologia, Departamento de Ciências Naturais, Universidade Estadual do Sudoeste da Bahia - UESB, Caixa Postal 95, 45083-900 Vitória da Conquista (BA). E-mail: raquelperezmaluf@gmail.com (*) Autora correspondente.

$\left({ }^{4}\right)$ Laboratório de Entomologia, Departamento de Fitotecnia e Zootecnia, Universidade Estadual do Sudoeste da Bahia - UESB, Vitória da Conquista (BA). 


\section{Introdução}

A fruticultura vem ganhando destaque especial na economia brasileira com a adaptação de inúmeras frutas às condições do solo e clima, tornando-se o país um dos maiores produtores mundiais de frutas (RAmos et al., 2001). Dentre as frutas tropicais brasileiras, a goiaba (Psidium guajava) tem lugar de destaque, não só pelo seu sabor como também pelo seu valor nutricional, sendo o Brasil o maior produtor de goiabas vermelhas (FRANCISCO et al., 2005). A grande perecidade da fruta e a produção de frutos sem os padrões estabelecidos no mercado internacional são fatores que dificultam o aumento da exportação da goiaba (GoNZAGA-NETO e SOARES, 1994).

Mesmo em culturas frutíferas em que a autofecundação é predominante, como na goiaba (MANICA et al., 2000), na presença de visitantes florais que promovam a polinização cruzada é favorecido o incremento na produção de frutos (Alves e Freitas, 2007), além de sua uniformidade e qualidade. Gonzaga-Neto e Soares (1994) relatam Apis mellifera como principal inseto polinizador das flores. A maior freqüência de $A$. mellifera nessa cultura foi verificada por Boti (2001), no Espírito Santo. Siqueira et al. (2005), no submédio do Vale do São Francisco, constataram serem as abelhas dos gêneros Centris e Xylocopa os polinizadores efetivos da goiaba. Em revisão feita por HEARD (1999), abelhas sem ferrão foram observadas coletando pólen nas flores de goiaba na Guatemala e Costa Rica. Na mesma região também foi constatada a presença das abelhas $A$. mellifera e as dos gêneros Bombus, Lasioglossum e Xylocopa.

Nos trabalhos constatam-se diferenças na composição e na importância das abelhas visitantes e prováveis polinizadoras da cultura da goiaba. A realização de estudos em diferentes regiões de cultivo dessa frutífera é interessante e, assim, o objetivo deste trabalho foi o de realizar um primeiro estudo sobre a diversidade de abelhas visitantes das flores da goiabeira, em pomares comerciais de Salinas (MG).

\section{Material e Métodos}

O trabalho foi desenvolvido na Fazenda Experimental Santa Isabel, município de Salinas (MG), em uma área de 1 ha, cultivada com goiaba cultivar Paluma, na florada induzida de maio de 2005. Na época, a cultura estava com quatro anos e meio de idade, altura média dos arbustos de $2,0 \mathrm{~m}$, no espaçamento $6,0 \times 5,0 \mathrm{~m}$, totalizando 330 plantas. $\mathrm{O}$ pomar está limitado ao Norte por um plantio de tangerinas, ao Sul por um de coco anão, a Leste, canade-açúcar e a Oeste, reserva de vegetação caatinga.
As coletas de abelhas foram realizadas com o auxílio de três coletores nos horários de $6 \mathrm{~h}$ às $18 \mathrm{~h}$ em seis faixas de horário (FX): 6h-8h; 8h-10h; 10h-12h; 12h-14h; 14h-16h e 16h-18h, totalizando 44 horas de coleta. As abelhas foram identificadas pelo Dr. Fernando Amaral da Silveira e depositadas na Coleção de Abelhas da UESB.

Para o estudo da estrutura da comunidade de abelhas visitantes das flores da goiaba foram estimados a riqueza de espécies (S), o índice de diversidade de Shannon $\left(\mathrm{H}^{\prime}\right)$, eqüitatividade $(\mathrm{J})$, a freqüência relativa (calculada a partir do número total de indivíduos da espécie i em relação ao total de indivíduos coletados), a constância (calculada a partir do total de coletas contendo a espécie i em relação ao total de coletas. Classificam-se as espécies em constantes $-W$, quando presentes em mais de $50 \%$ das coletas; em acessórias $-Y$, quando presentes entre $25 \%$ e $50 \%$ das coletas e em acidentais - $Z$, quando presentes em menos de $25 \%$ das coletas), e as espécies dominantes, quando as freqüências relativas fossem superiores a 1/S (Silveira Neto et al.,1976).

Foram realizadas análises de correlação de Pearson entre os fatores abióticos - temperatura máxima, temperatura mínima, temperatura média e umidade relativa $12 \mathrm{~h}$, e número de indivíduos coletados. Os dados meteorológicos foram obtidos na estação meteorológica de Salinas (MG).

Para avaliação da influência do horário de amostragem no total de abelhas coletadas foi feita uma análise não paramétrica, pelo teste Kruskal-Wallis $(\mathrm{H})$.

\section{Resultados e Discussão}

Foram coletadas 705 abelhas, pertencentes a 17 espécies. Houve predominância de 16 espécies nativas brasileiras com 690 indivíduos, em relação a A. mellifera, com apenas 15 indivíduos (Tabela 1). Considerando-se o total de abelhas coletadas no pomar de goiaba, a espécie mais abundante e dominante foi Trigona spinipes sugerindo, pela sua presença constante nas flores da goiaba, um provável papel polinizador no pomar avaliado. As demais espécies foram coletadas de maneira acidental, com exceção de Melipona quadrifasciata, A. mellifera e Tetragonisca angustula, consideradas acessórias (Tabela1). Este resultado é divergente dos trabalhos realizados na cultura da goiaba em que $A$. mellifera é a espécie mais freqüente em diferentes regiões do Brasil como na Bahia e Espírito Santo (CASTRO e Araújo, 1998, citados por CASTro, 2002; Boti, 2001). Воті (2001) considerou as espécies Epicharis flava, Eulaema nigrita, Augochloropsis patens, C. tarsata, 
Euglossa sp., Oxaea flavescens, Oxytrigona sp., Xylocopa frontalis e Bombus morio potenciais polinizadoras da goiaba, devido ao comportamento das abelhas nas flores, com favorecimento da transferência do grão de pólen do corpo das abelhas para o estigma das flores. SIQUEIRA et al. (2005), no submédio do Vale do São Francisco, constataram serem as abelhas dos gêneros Centris e Xylocopa os polinizadores efetivos da goiaba. Em Salinas, essas espécies, quando coletadas, não o foram de maneira expressiva (Tabela 1).

Tabela 1. Número de indivíduos $(\mathrm{N})$, freqüência relativa $(\mathrm{F})$, constância $(\mathrm{C})$ e dominância (D) de Apoidea, em pomar comercial de goiaba (Psidium guajava). Salinas (MG), maio de 2005

\begin{tabular}{|c|c|c|c|c|c|}
\hline Família & Espécie & $\mathrm{N}$ & $\mathrm{F}$ & $\mathrm{C}^{1}$ & $\mathrm{D}^{2}$ \\
\hline \multirow[t]{7}{*}{ Andrenidae } & Oxaea cfr. austera & 2 & 0,0028 & $\mathrm{Z}$ & $\mathrm{Nd}$ \\
\hline & Oxaea flavescens & 4 & 0,0057 & $\mathrm{Z}$ & $\mathrm{Nd}$ \\
\hline & Apis mellifera & 15 & 0,0213 & Y & $\mathrm{Nd}$ \\
\hline & Bombus (Fervidobombus) morio & 6 & 0,0085 & $\mathrm{Z}$ & $\mathrm{Nd}$ \\
\hline & Epicharis (Epicharana) flava & 3 & 0,0043 & $\mathrm{Z}$ & $\mathrm{Nd}$ \\
\hline & Euglossa sp1 & 1 & 0,0014 & Z & $\mathrm{Nd}$ \\
\hline & Euglossa sp2 & 5 & 0,0071 & $\mathrm{Z}$ & $\mathrm{Nd}$ \\
\hline \multirow[t]{8}{*}{ Apidae } & Exomalopsis (Exomalopsis) analis & 1 & 0,0014 & Z & $\mathrm{Nd}$ \\
\hline & Melipona quadrifasciata & 42 & 0,0596 & Y & $\mathrm{D}$ \\
\hline & Melitoma segmentaria & 1 & 0,0014 & $\mathrm{Z}$ & $\mathrm{Nd}$ \\
\hline & Tetragonisca angustula & 16 & 0,0227 & $\mathrm{Y}$ & $\mathrm{Nd}$ \\
\hline & Trigona spinipes & 600 & 0,8511 & W & $\mathrm{D}$ \\
\hline & Xylocopa (Neoxylocopa) frontalis & 4 & 0,0057 & $\mathrm{Z}$ & $\mathrm{Nd}$ \\
\hline & Xylocopa (Neoxylocopa) grisescens & 1 & 0,0014 & $\mathrm{Z}$ & $\mathrm{Nd}$ \\
\hline & Augochlora sp & 1 & 0,0014 & $\mathrm{Z}$ & $\mathrm{Nd}$ \\
\hline \multirow[t]{2}{*}{ Halictidae } & Augochloropsis sp1 & 2 & 0,0028 & $\mathrm{Z}$ & $\mathrm{Nd}$ \\
\hline & Augochloropsis sp2 & 1 & 0,0014 & $\mathrm{Z}$ & $\mathrm{Nd}$ \\
\hline
\end{tabular}

(1) W: constante. Y: acessória. Z: acidental.

$\left({ }^{2}\right)$ D: dominante. Nd: não dominante.

As abelhas foram coletadas predominantemente no período matutino, de $6 \mathrm{~h}$ às $10 \mathrm{~h}$, correspondendo às faixas FX1 e FX2 (Tabela 2), quando aproximadamente $84 \%$ dos indivíduos foram coletados $(\mathrm{H}=15,88 ; \mathrm{P}=0,007)$. A partir das $6 \mathrm{~h}$, as flores já estavam abertas, sendo favorecida a atratividade dos visitantes florais. O pólen parece ser o único recurso oferecido por essa cultura (Siqueira et al., 2005) e deve estar disponível em mais quantidade durante esse período, visto que foram constatadas abelhas forrageando em outras espécies vegetais próximas às goiabeiras no período da tarde. Essa observação de mais atividade das abelhas nos horários da manhã tem uma conseqüência prática para o manejo da cultura da goiaba na região de Salinas, recomendando-se a aplicação de agrotóxicos e outras práticas de interferência no comportamento das abelhas nas flores preferencialmente na parte da tarde, para não haver prejuízos à polinização e conseqüentemente à produção de frutos.

Quanto aos dados abióticos avaliados, temperatura média, mínima e máxima e umidade relativa do ar, não houve influência significativa na atividade das abelhas nas flores de goiaba ao longo do período de amostragem, como pode ser verificado pelos valores estimados pela correlação de Pearson:

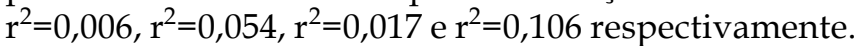

A diversidade estimada pelo índice de Shannon foi correspondente a 0,72 e o índice de eqüitatividade foi 0,25 . Esses índices são reflexo de uma reduzida diversidade e da dominância de uma espécie em relação ao total de indivíduos coletados, no caso T. spinipes. Em outros trabalhos realizados com abelhas em culturas agrícolas foram determinados índices similares. Na cultura do urucum, segundo CostA et al. (2008), a diversidade foi de 1,58 e a eqüitatividade 0,51 . Em Lavras (MG), de acordo com Morgado et al. (2002), em fevereiro, os índices de diversidade e eqüitatividade nas flores de girassol foram, respectivamente, 1,56 e 0,36. Em junho, na mesma cidade e nas mesmas flores, foi verificada uma diferença no índice de diversidade, 3,07, e mais diversidade de espécies. 
Tabela 2. Abundância das espécies de abelhas coletadas em flores de goiaba (Psidium guajava) em relação aos horários amostrados. Salinas (MG), maio, 2005.

\begin{tabular}{|c|c|c|c|c|c|c|}
\hline \multirow{2}{*}{ Espécie } & \multicolumn{6}{|c|}{ Horário de Amostragem } \\
\hline & FX1 & FX2 & FX3 & FX4 & FX5 & FX6 \\
\hline Oxaea cfr. austera & - & 1 & - & - & 1 & - \\
\hline Oxaea flavescens & 2 & - & 1 & 1 & - & - \\
\hline Apis mellifera & 8 & 4 & - & - & 2 & 1 \\
\hline Bombus morio & 1 & 5 & - & - & - & - \\
\hline Epicharis flava & - & 1 & 1 & - & 1 & - \\
\hline Euglossa sp1 & - & - & 1 & - & - & - \\
\hline Euglossa sp2 & - & 2 & 3 & - & - & - \\
\hline Exomalopsis analis & - & - & 1 & - & - & - \\
\hline Melipona quadrifasciata & 36 & 6 & - & - & - & - \\
\hline Melitoma segmentaria & - & 1 & - & - & - & - \\
\hline Tetragonisca angustula & - & 5 & 7 & 1 & 2 & 1 \\
\hline Trigona spinipes & 230 & 289 & 77 & - & 3 & 1 \\
\hline Xylocopa frontalis & - & 2 & 1 & 1 & - & - \\
\hline Xylocopa grisescens & - & 1 & - & - & - & - \\
\hline Augochlora sp & - & - & - & 1 & - & - \\
\hline Augochloropsis sp1 & - & - & - & 2 & - & - \\
\hline Augochloropsis sp2 & - & - & 1 & - & - & - \\
\hline Total & 277 & 317 & 93 & 6 & 9 & 3 \\
\hline
\end{tabular}

FX1:6h-8h; FX2: 8h-10h; FX3: 10h-12h; FX4:12h-14h; FX5:14h-16h; FX6:16h-18h

A abundância e a distribuição de espécies de abelhas polinizadoras são dependentes da disponibilidade de flores para forrageamento e de locais de nidificação (Willians, 2002). Bоті (2001) constatou mais riqueza e abundância de abelhas em pomares de goiaba próximos às áreas de fragmentos de mata nativa, em distância inferior a $300 \mathrm{~m}$, quando comparados aos pomares localizados mais distantemente de fragmentos, em distância superior a $1.500 \mathrm{~m}$; além disso, nos pomares próximos aos fragmentos foi determinada maior taxa de frutificação. Com a fragmentação e a descontinuidade do habitat, provocadas por grandes extensões de áreas cultivadas, tem-se um declínio nas populações de abelhas nativas (Willians, 2002; Westerkamp e GotTsberger, 2000). Assim, a presença de áreas nativas preservadas deve ser favorecida nas culturas de frutíferas. Na Fazenda Santa Isabel, os pomares são limítrofes às áreas de caatinga, o que poderá garantir a manutenção das espécies polinizadoras ao longo das estações.

\section{Referências}

ALVES, J.E.; FREITAS, B. M. Requerimento de polinização da goiabeira. Ciência Rural, Santa Maria, v.37, n.5, p.12811286, 2007.
BOTI, J.B. Polinização entomófila da goiabeira (Psidium guajava L., Myrtaceae): influência da distância de fragmentos florestais em Santa Teresa, Espírito Santo. 2001. 57f. Dissertação (Mestrado em Entomologia) - Universidade Federal de Viçosa, Viçosa, 2001.

CASTRO, M.S. Bee fauna of some tropical and exotic fruits: potencial pollinators and their conservation. In: KEVAN, P.; IMPERATRIZ FONSECA, V.L. (Ed.). Pollinating Bees: The conservation link between agriculture and nature. Brasília: Ministério do Meio Ambiente, 2002. p.275-288.

COSTA, A.J.C; GUIMARÃES-DIAS, F.; PÉREZ-MALUF, R. Abelhas visitantes das flores de urucum (Bixa orellana $\mathrm{L}$.), em Vitória da Conquista-BA. Ciência Rural, Santa Maria, v.38, n.2, p.534-537, 2008.

FRANCISCO, V.L.F.S.; BAPTISTELLA, C.S.L.; AMARO, A.A. A cultura da goiaba em São Paulo. 2005. Disponível em: <http:/ /www.iea.sp.gov.br>. Acesso em 06/10/2006.

GONZAGA-NETO, L.; SOARES, J.M. FRUPEX. Goiaba para exportação. Aspectos técnicos da produção. Brasília: Embrapa, 1994. 49p.

HEARD, T. A. The role of stingless bees in crop pollination. Annual Review of Entomology, Queensland, v.44, p.183206, 1999. 
MANICA, I.; ICUMA, I.M.; JUNQUEIRA, M.T.V.;SALVADOR, J.O.; MOREIRA, A.; MALAVOLTA, E. Fruticultura Tropical 6. Porto Alegre: Cinco Continente, 2000. 374p.

MORGADO, L. N.; CARVALHO, C. F.;SOUZA, B.;SANTANA, M. P. Fauna de abelhas (Himenoptera: Apoidea) nas flores de Girassol Helianthus annuus L., em Lavras, MG. Ciência e Agrotecnologia, Lavras, v.26, n.6, p.1167 - 1177, 2002.

RAMOS, J.D.; PASQUAL, M.; CHALFUN, N.N.J. Fruticultura Tropical. Introdução, situação e perspectivas. Lavras: FAEPE, 2001. 72p.

SILVEIRA NETO, S.; NAKANO, O.; VILA NOVA, N. A. Manual de ecologia dos insetos. Piracicaba: Ceres, 1976. 419 p.

SIQUEIRA, K.M.M.; MARTIN, C.F.; SCHLINDWEIN, C.; KILL, L.H.P. Polinizadores efetivos da goiaba (Psidium guajava) em área irrigada do submédio do Vale do São Francisco. In: ENCONTRO DE ZOOLOGIA DE NORDESTE, 15., 2005, Salvador. Resumos... Salvador: Universidade do Estado da Bahia, 2005. p.260.

WILLIANS, I. Insect pollination and crop production: an European perspective. In: KEVAN, P. e IMPERATRIZ FONSECA, V.L. (Ed.). Pollinating Bees: The conservation link between agriculture and nature. Brasília: Ministério do Meio Ambiente, 2002. p.59-65.

WESTWERKAMP, C.; GOTTSBERGER, G. Diversity pays in crop pollination. Crop Science, Denver, v.40, n.5, p.1209-1222, 2000. 